\section{Nanoceramics in Biomedical Applications}

\author{
B. Ben-Nissan
}

\begin{abstract}
An improved understanding of the interactions at the nanoscale level between the bioceramics in medical implants and the hard or soft tissues in the human body could contribute significantly to the design of new-generation prostheses and postoperative patient management strategies. Overall, the benefits of advanced ceramic materials in biomedical applications have been universally accepted, specifically in terms of their strength, biocompatibility, hydrophilicity, and wear resistance in articulating joints. The continuous development of new-generation implants utilizing nanocoatings with novel nanosensors and devices is leading to better compatibility with human tissue and improved well-being and longevity for patients. This article gives a short overview of bioceramics and reexamines key issues of concern for processing and applying nanoceramics as biomaterials.
\end{abstract}

Keywords: bioceramics, biomaterials, implants, nanoceramics, nanostructure.

\section{Introduction}

At present, the most common materials in clinical use are those chosen from a handful of well-characterized and available biocompatible ceramics, metals, polymers, and their combinations as composites or hybrids. Advances in the fundamental understanding of cell and molecular biology, tissue engineering, targeted drug delivery, wound healing, and other biomedical processes, together with the development of new enabling technologies such as microscale, nanoscale, and bio-inspired fabrication (biomimetics) and surface modification methods, have the potential to drive at an unprecedented rate the design and development of new biomaterials useful for medical applications. The current focus is on the production of new nanoceramics relevant to a broad range of applications such as implantable surfacemodified medical devices for better hardand soft-tissue attachment, increased bioactivity, tissue regeneration and engineering, cancer treatment, drug and gene delivery, treatment of bacterial and viral infections, delivery of oxygen to damaged tissues, imaging, and materials for mini- mally invasive surgery. A more futuristic view includes nanorobotics, nanobiosensors, and micro-nanodevices for a wide range of biomedical applications.

The opportunity to significantly improve the clinical usefulness of biomaterials, particularly on the nanoscale, provided by these advances should be seized by promoting and supporting research and development of novel new-generation biomaterials with improved bioactivity and mechanical properties. Specifically, these new concepts and strategies for fabrication methods and improved tissue interactions could lead to biomaterials that are truly biocompatible and bioresponsive at a nanoscale level, similar to biogenic (natural) materials.

A biomaterial by definition is a nondrug substance suitable for inclusion in systems that augment or replace the function of bodily tissues or organs. A century ago, artificial devices made from materials as diverse as gold and wood were developed to a point where they could replace various components of the human body. These materials are capable of being in contact with body fluids and tissues for prolonged periods of time while eliciting little if any adverse reaction.

The main factors in any biomaterial's clinical success are its biocompatibility and biofunctionality, which are directly related to tissue/implant interface interactions. Improvement of interface bonding by nanoscale coatings, based on biomimetics, has been of worldwide interest during the last decade. Currently, a number of companies are in the commercialization stages of new-generation nanoscalemodified implants for orthopedic, ocular, and maxillofacial surgery and for hardand soft-tissue engineering (e.g., IsoTis BV, ApaTech Ltd., Etex Corp.).

The worldwide biomaterials market is valued at close to $\$ 24$ billion. Orthopedic and dental applications represent approximately $55 \%$ of the total biomaterials market. Sales of orthopedic products worldwide exceeded $\$ 13$ billion in 2000 an increase of $12 \%$ over 1999 revenues. ${ }^{2}$ Expansion in these areas is expected to continue, due to a number of factors including the aging population, an increasing preference by younger to middle-aged candidates to undergo surgery, improvements in technology, a better understanding of biomechanics, the desire to improve one's appearance, and the demand by patients for better performance from orthopedic products.

\section{Biomaterials and Bioceramics}

It has been long accepted that no foreign. material placed within a living body is completely compatible with it. The only substances that conform completely are those manufactured by the body itself (autogenous); any other substance that is recognized as foreign initiates some type of reaction. The question for biomaterials researchers is, with our ability to control surfaces at the nanoscale level, and with the range of new-generation novel "nanotreatments" being developed, can we somehow deceive the body into allowing synthetic materials to be accepted as biogenic or autogenic?

When a synthetic material is implanted in the human body, the tissue reacts in a variety of ways, depending on the material used. The mechanism of tissue interaction at the nanoscale depends on the tissue's response to the implant surface. In general, three terms describe or classify a biomaterial with respect to the tissue response: bioinert, bioresorbable, and bioactive. A bioinert material, such as alumina, is accepted by the body but does not interact or react with the physiological environment; a bioresorbable material, such as 
a bioglass, is surface-reactive and dissolves within the physiologic environment to be replaced by the soft or hard tissue; and a bioactive material, such as hydroxyapatite, reacts with the tissue and generates good chemical bonding. These types, each allowing different means of achieving attachment of implants to the musculoskeletal system, are covered in a range of comprehensive excellent reviews. ${ }^{3-6}$

In the early 1970s, bioceramics were employed in singular, biologically inert applications such as bone replacement. Given that cells and tissues in the body perform many other vital regulatory and metabolic functions, the role of bioceramics has changed from maintaining an essentially physical function, without eliciting a host response, to providing a more integrated interaction with the host. This has been accompanied by an increasing demand on medical devices to improve the quality of life as well as to extend its duration.

\section{Bone Replacement Applications}

Nanotechnology has opened novel techniques for the production of bonelike synthetic nanopowders and coatings of hydroxyapatite (HAp). Nanoparticles of $\mathrm{HAp}\left[\mathrm{Ca}_{10}\left(\mathrm{PO}_{4}\right)_{6}(\mathrm{OH})_{2}\right]$ have generated new opportunities in designing superior biocompatible coatings for implants and in developing high-strength orthopedic and dental nanocomposites. Various companies have synthesized HAp nanoparticles with diameters in the range of $15-20 \mathrm{~nm}$ and HAp coatings $40 \mathrm{~nm}$ thick. Nanoparticles and nanoplatelets of HAp provide excellent bioactivity arising from the very high surface area for integration into bone (Figure 1). Some production techniques and surface modifications (e.g., some of the sol-gel techniques, chemical and vapor deposition techriques, plasma spray) result in bonds with excellent ad-

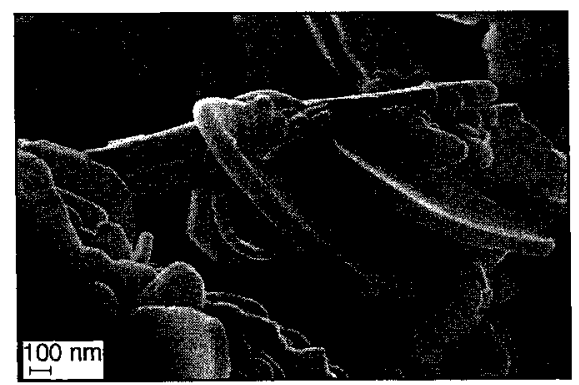

Figure 1. Scanning electron microscopy image of bonelike carbonated apatite platelets produced at $300^{\circ} \mathrm{C}$ from a sol-gel alkoxide system (image by $B$. Ben-Nissan, A. Milev, and R. Wuhrer). hesive strength between the HAp and the substrate materials, while others are poor (electrophoretic deposition, various solution dip-coating systems, thermal spray). Currently, a number of companies are producing nanocomposites (e.g., Mitsubishi Materials, ApaTech Ltd., Dentsply International, Interpore Cross International, Wyeth BioPharma, and Medtronic Sofamor Danek) that incorporate macro- and nanoparticles of HAp and organic and biogenic materials (e.g., polyethylene, synthetic peptides and collagen, growth factors). The combination of macro- and nanoparticles provides mechanical strength not achievable using nanoparticles alone. With some of the materials, enhanced bioactivity and mechanical properties have been reported in orthopedic and dental applications such as bone cements and dental fillings. ${ }^{7}$

The bone mineral is composed of nanocrystals-or, more accurately, nanoplatelets-originally described as hydroxyapatite and similar to the mineral dahllite. It is now agreed that bone apatite can be better described as carbonate hydroxyapatite $(\mathrm{CHA})$ and approximated by the formula $(\mathrm{Ca}, \mathrm{Mg}, \mathrm{Na})_{10}\left(\mathrm{PO}_{4}, \mathrm{CO}_{3}\right)_{6}(\mathrm{OH})_{2}$. The composition of commercial CHA is similar to bone mineral apatite. Bone pore sizes range from $1 \mu \mathrm{m}$ to $100 \mu \mathrm{m}$ in normal cortical bone and from $200 \mu \mathrm{m}$ to $400 \mu \mathrm{m}$ in trabecular bone tissue (found in the ends of long bones), and the pores are interconnected. Porosity (macroporosity) is introduced in synthetic calcium phosphates [HAp, tricalcium phosphate $(\beta-\mathrm{TCP})$, $\mathrm{CHA}]$ by the addition of volatile compounds such as naphthalene or hydrogen peroxide.

Bonelike HAp nanopowders and nanoplatelets can be synthesized by a range of production methods; ${ }^{8}$ however, one very promising method is synthesis from solgel aqueous solutions. Early published work shows that while biphasic sol-gel HAp products are easily synthesized, monophasic HAp powders and coatings were more difficult to produce. For example, synthesis from a stoichiometric ratio of mixed salt alkoxide sol-gel systems produces impure HAp accompanied by a second phase of calcium oxide. Further investigations into the process showed that the time period between the mixing of precursors and heating to remove the solvent ("aging time") can significantly alter the composition of the product. ${ }^{8}$

Orthopedic implants used mainly for joint replacement and fracture fixation include metallic (cobalt chromium or titanium alloys) implants, screws, plates, and nails and their various permutations and combinations. The most important pa- rameters for these implants are that they have the necessary wear resistance, allow for adequate attachment to bone, and display the required strength, ductility, and elasticity. ${ }^{7}$ At a bone-implant load-carrying interface, the greater the implant material stiffness, the greater load it carries, compared with the surrounding tissue. This imbalance in load, known as stress shielding, can cause bone tissue to be resorbed. An implant that is too rigid may also increase the likelihood of bone fracture, as the bone becomes osteoporotic (thinned) due to the excessive protection generated by the stress-shielding effect of the implant. Implants nanocoated with calcium phosphate and possessing the appropriate bioactivity characteristics, bonding ability, and design may be the answer to this serious problem. Oonishi et al. ${ }^{7}$ reported in their experimental and long-term clinical experience that bioceramics, including bioinert and bioactive ceramics, are and will be playing a major role in the longevity of total joint replacements.

\section{Calcium Phosphate Coatings}

It has been accepted that porous HAp cannot be used for load-bearing applications due to its unfavorable mechanical properties. For this reason, HAp has been used instead as a thin-film coating on metallic alloys in orthopedic surgery. Of the metallic alloys used, cobalt chromium alloys and titanium-based alloys are the preferred materials for these thin-film HAp coatings.

Thermal spraying tends to be the most commonly used coating technique, but in clinical situations, it is difficult to produce a controllable resorption response using implants coated by this method. Problems arise due to compositional changes in HAp during spraying at $1600^{\circ} \mathrm{C}$ and uneven distribution of the sprayed material, resulting in gaps. Other techniques that are capable of producing thin coatings include pulsed laser deposition and sputtering, which, like thermal spraying, involves high-temperature processing. Other techniques capable of creating nanocoatings, such as electrodeposition and sol-gel, use lower temperatures and thus avoid the problems associated with the structural instability of HAp at elevated temperatures.

The advantages of the sol-gel technique are numerous; it is nanoscale; it results in a stoichiometric, homogeneous, and pure coating due to mixing on the molecular scale; it allows reduced firing temperatures due to its small particle sizes with high surface areas; it has the ability to produce uniform fine-grained structures; it allows the use of different chemical routes (alkoxide or aqueous-based); and it is easily 
applied to complex shapes with a range of coating techniques including dip, spin, and spray coating. Currently, $40 \mathrm{~nm}$ singlelayer pure $\mathrm{HAp}$ or carbonated apatites are easily produced. Multilayered coatings of up to 12 layers have been reported. ${ }^{8}$

Developing an understanding of new materials at the molecular and nanoscale level has become increasingly critical in the new era of biomedical materials for the design and synthesis of nanodevices on the molecular scale. New technologies, including molecular self-assembly as a fabrication tool, will become increasingly more important in the coming decades.

Basic engineering principles for microfabrication can be learned through understanding the phenomenon of molecular self-assembly, which is ubiquitous in the natural world. Coral and bone formation are good examples of these biomimetic processes. Coralline apatites can be derived from sea coral, which is composed of calcium carbonate in the form of aragonite and is a naturally occurring material that has optimal strength and structural characteristics. The pore structure of coralline calcium phosphate produced by certain species is similar to human cancellous bone, making it a suitable material for bone-graft applications. An Australian coral was converted to monophasic HAp using a two-stage process in which the hydrothermal conversion method was followed by a patented HAp sol-gel nanocoating 9 process. This nanocoating was reported to produce a twofold increase in the biaxial strength of the converted and nanocoated coral, as compared with the converted-only form. Addition of bone morphogenic proteins (BMP-2), growth factors, collagen, and stem cells to these and similar scaffolding materials is one of the ongoing research areas in the nanoceramics field.

\section{Simulated Body Fluid (SBF)}

Nanoscale coatings and surface modification methods are currently being used to produce body-interactive materials, helping the body to heal, and promoting regeneration of tissues, thus restoring physiological function. This approach is being explored in the development of a new generation of nanobioceramics with a widened range of applications in maxillofacial, orthopedic, and ocular surgery.

Hydroxyapatite layers can be easily produced on various organic or inorganic substrates in simulated body fluid (SBF). SBF has ionic concentrations of $142.0 \mathrm{mM}$ $\mathrm{Na}^{+}, 5.0 \mathrm{mM} \mathrm{K}+, 1.5 \mathrm{mM} \mathrm{Mg}{ }^{2+}, 2.5 \mathrm{mM}$ $\mathrm{Ca}^{2+}, 147.8 \mathrm{mM} \mathrm{Cl}, 4.2 \mathrm{mM} \mathrm{HCO}_{3}^{-}$, $1.0 \mathrm{mM} \mathrm{HPO}{ }^{2-}$, and $0.5 \mathrm{mM} \mathrm{SO}{ }^{2-}$. Kokubo $^{10}$ showed that after immersion in
SBF (see Figure 2), a wide range of biomaterial surfaces display very fine crystallites of carbonate-ion-containing apatite. ${ }^{11}$ Bone cells have been shown to proliferate and differentiate on this apatite layer, showing increased bioactivity and new bone formation.

This SBF is highly supersaturated in calcium and phosphate with respect to apatite under even normal conditions. Therefore, if a material has an effective functional group for apatite nucleation on its surface, it can form the apatite spontaneously. It has been reported ${ }^{10}$ that this nucleation rate can be increased if excessive amounts of $\mathrm{Ca}^{2+}$ ions, $\mathrm{PO}_{4} \mathrm{H}_{2}, \mathrm{Si}-\mathrm{OH}, \mathrm{Ti}-\mathrm{OH}, \mathrm{Zr}-\mathrm{OH}$, $\mathrm{Ta}-\mathrm{OH}, \mathrm{Nb}-\mathrm{OH}$, or similar functional groups are present. It has been shown ${ }^{\text {I }}$ that highly porous gels of $\mathrm{SiO}_{2}, \mathrm{TiO}_{2}, \mathrm{ZrO}_{2}$, $\mathrm{Nb}_{2} \mathrm{O}_{5}$, and $\mathrm{Ta}_{2} \mathrm{O}_{5}$ could form nanocrystalline apatite layers on their surfaces in SBF solutions.

This method is also being used to measure the level of bioactivity of an artificial material by examining apatite-forming ability on its surface.

\section{Nano- and Macrobioceramics for Radiotherapy}

In drug delivery, the primary aim is to target drugs to specific sites within the body. Various nanoceramic drug delivery systems are currently in clinical evaluation stages. In addition to reducing toxicity to nondiseased cells, the use of these nanoceramic systems has the potential benefit of increasing drug efficiency, which translates to significant cost savings for many of the expensive drug treatments now being engineered. Nano drug delivery systems also have the extraordinary characteristic of being able to target and control drug release with very high precision (see the article by Kriven et al. in this issue of MRS Bulletin).

Despite exhibiting excellent preclinical potentials, several anticancer drugs fail in their desired clinical activity due to the lack of specific targeted delivery. Nanoceramics are expected to be instrumental in achieving this targeted delivery.

One of the most common approaches in cancer treatment is the removal of the diseased parts; unfortunately, recovery or return of full function is seldom achieved with this approach. Non-invasive treatment techniques, in which only the cancer cells are destroyed, were introduced in mid$1980 \mathrm{~s}^{12}$ Glass microspheres of $17 \mathrm{Y}_{2} \mathrm{O}_{3}$ $19 \mathrm{Al}_{2} \mathrm{O}_{3}-64 \mathrm{SiO}_{2}$ (mol\%) composition, 20-30 $\mu \mathrm{m}$ in diameter, were shown to be effective for targeted radiotherapy of liver cancer. ${ }^{10,13}$ The ${ }^{89} Y$ in this glass is nonradioactive, but can be activated by neutron bombardment to ${ }^{90} \mathrm{Y}$, which is a $\beta$-emitter with a half-life of $64.1 \mathrm{~h}$. Current application involves injection of these spheres into a diseased liver through the hepatic artery, where they are entrapped in small blood vessels, blocking the blood supply to the cancer and directly irradiating the cancer with $\beta$ rays. These glass microspheres are already in clinical use in Australia, Canada, and the United States.

Oxygen is known to be poorly supplied to the interior of a tumor because it cannot penetrate the surrounding layers of cancerous cells, and hence a tumor can be destroyed at temperatures of around $43^{\circ} \mathrm{C}$, whereas normal cells can be kept alive even around $48^{\circ} \mathrm{C}$. If ferri- or ferromagnetic materials are implanted around cancers and placed under an alternating magnetic field, it is expected that cancer cells that are locally heated can be destroyed by the magnetic hysteresis loss of the ferri- or ferromagnetic materials. In ceramics, a critical pore and grain size can be varied from a few nanometers up to several tens of nanometers to control the ease of delivery and dispersion of the materials to the targeted area. Thus, the characteristic size of the domain plays a key role in nanostructured ferromagnetic and ferroelectric materials. An extremely high magnitude of magnetic and dielectric permittivity and the transition in a paramagnetic or paraelectric state can be observed by decreasing grain size down to the nanometer scale. The use of these nano-sized materials is currently being investigated for cancer treatment applications.

Kokubo and co-workers ${ }^{13,14}$ prepared a ferrimagnetic glass ceramic containing $36 \mathrm{wt} \%$ magnetite $\left(\mathrm{Fe}_{3} \mathrm{O}_{4}\right), 200 \mathrm{~nm}$ in diameter, in a CaO-SiO ${ }_{2}$ matrix. It was reported that cancerous cells in the medullary (internal) canal of rabbit tibia were completely destroyed when this glass ceramic was inserted into the tibia and placed under an alternating magnetic field of $300 \mathrm{Oe}$ at $100 \mathrm{kHz} \cdot{ }^{13,14}$ This kind of invasive treatment, however, cannot be applied to humans, since cancer cells metastasize. In human applications, ferri- or ferromagnetic material must be delivered to the cancer by injection through blood vessels, similar to the radioactive microspheres. For this purpose, the heat-generating efficiency of the ferrimagnetic material must be further increased. Recently, microspheres $20-30 \mu \mathrm{m}$ in diameter were formed by depositing $50 \mathrm{~nm}$ magnetite particles on silica microspheres $12 \mu \mathrm{m}$ in diameter, through the deposition of $\mathrm{FeOOH}$ in a solution and its subsequent transformation into $\mathrm{Fe}_{3} \mathrm{O}_{4}$ at $600^{\circ} \mathrm{C}$ under $\mathrm{a} \mathrm{CO}_{2}-\mathrm{H}_{2}$ gas atmosphere. ${ }^{14}$ It was reported that its heatgenerating efficiency was about four times that of the glass ceramic. 


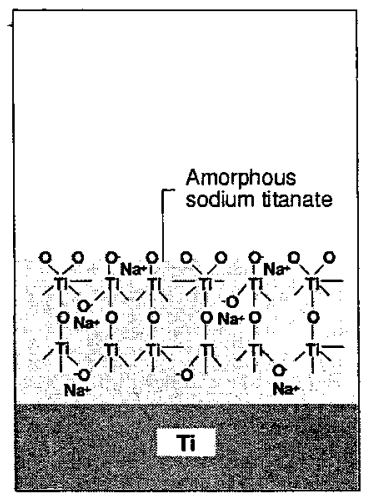

Before soaking in SBF

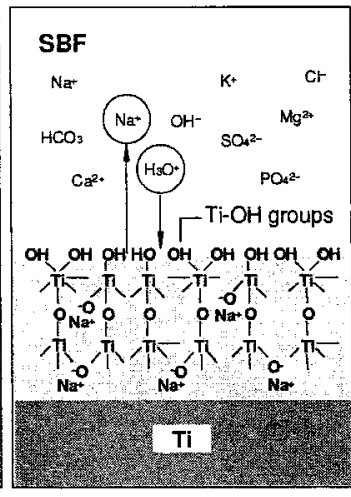

Formation of Ti-OH groups

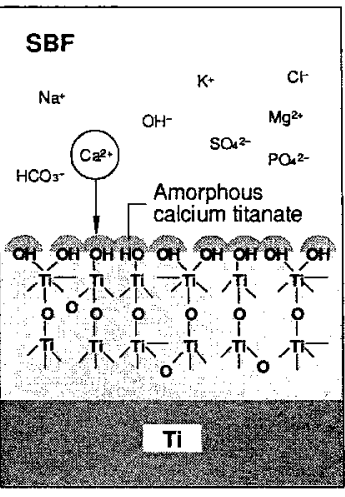

Formation of amorphous calcium titanate

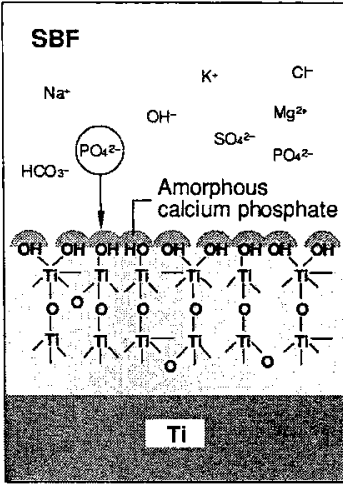

Formation of amorphous calcium phosphate

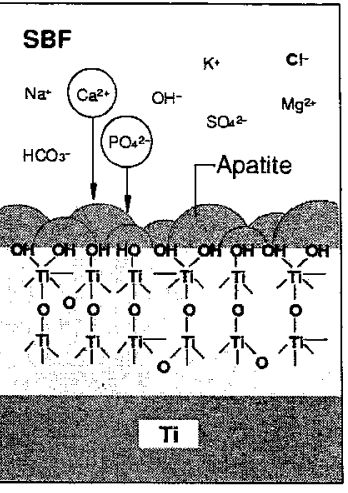

Formation of apatite Figure 2. Schematic representation of the mechanism of apatite formation on NaOH-treated Ti metal in simulated body fluid (SBF) (from
Reference 10).

The synthesis of nanoparticles using the restricted environments offered by surfactant systems such as water-in-oil microemulsions (reverse micelles) provides excellent control over particle size, interparticle spacing, and particle shape. The controlled environment of the reverse micelle also allows sequential synthesis, which can produce a core/shell structure. Interest in chemically functionalized nanoparticles has resulted in the fabrication of core/shell materials consisting of ferromagnetic nanoparticles with gold coatings. Other types of confined media, including microporous and mesoporous host materials, are used to fabricate particles with nanometer dimensions. The incorporation of nanophase magnetic particles into liposomes also promotes the development of materials with a variety of biomedical applications.

In a new nano-microemulsion system, it was shown that lipophilic drugs could be made soluble in the oil core of the microemulsion, hence preventing hydrolysis of the drug. For a controlled release of a drug at $43^{\circ} \mathrm{C}$, these microemulsion droplets can be coated with a polymer such as poly(lactic-co-glycolic acid). Due to their nanometer size (in the range of 40-60 nm) and biodegradable polymeric coating, these nanoencapsulates are expected to have a long circulation time within the body and can be released around a solid tumor by increasing the temperature to $43^{\circ} \mathrm{C}$ using a laser device.

\section{Bioengineering Applications}

At the micro/nano end of bioengineering, microtechnology and nanotechnology have been, and will increasingly be, used for the fabrication of biodevices meant for emerging applications such as a planar devices (e.g., biosensor arrays, DNA chips);

- micro/nano biodevices (e.g., nanoscale biosensors; large-scale integrated biosensors; dynamic equipment using protein molecular motors);

- artificial organs or tissues (e.g., neuronal cell networks);

- microfluidic devices and biochips (e.g., "lab on a chip");

- biomimetics engineering (e.g., biomimetic systems); and

- guided drug delivery to bio-MEMS (biorelated microelectromechanical systems).

Many of these "proof-of-concept" devices will be using new nanobioceramics in small quantities, a procedure similar to that currently used in the electronics industry, which still utilizes a range of advanced ceramics in small quantities to provide essential electronic properties.

\section{Tissue Engineering}

Recently, tissue engineering has been directed toward taking advantage of the combined use of living cells and threedimensional ceramic scaffolds to deliver vital cells to the damaged site in the body. Feasible and productive strategies have been aimed at combining a relatively traditional approach, such as bioceramic implants, with the acquired knowledge in the fields of cell growth and differentiation of osteogenic cells.

Stem cells have been incorporated into a range of bioceramics. A stem cell is a type of cell that has the ability to reproduce for a long period of time. It also can give rise to specialized cells that make up the tissues and organs of the body. An adult stem cell is an undifferentiated (unspecialized) cell that occurs in a differentiated (specialized) tissue, renews itself, and becomes specialized to yield all of the specific cell types of the tissue from which it originated. Cultured bone marrow cells, derived from adult stem cells, can be regarded as mesenchymal precursor cell populations and are similar to stem cells in that they also can differentiate into different lineages-osteoblasts, chondrocytes, adipocytes, and myocytes. When implanted into immunodeficient mice, these cells can combine with mineralized threedimensional scaffolds to form highly vascularized bone tissue. These nanoscale cultured cell/bioceramic composites can be used to treat full-thickness gaps in longbone shafts with excellent integration of the ceramic scaffold with bone and good functional recovery (see Figure 3). ${ }^{15}$ Excellent innovative work with these new nanobioceramics, including biogenic additives, is in progress, and clinical application is becoming common. ${ }^{16}$

\section{Concluding Remarks}

Nanotechnologies are one of the key advancements of the 21 st century. Rationally designed materials synthesized by "bottomup" approaches can permit the tailoring of properties from the atomic to the mesoscopic and macroscopic length scales. This ability to tailor a material's properties over broad length scales suggests that research on hybrids can have a significant impact on fields as diverse as nanophotonics, separation techniques, catalysis, smart coatings, sensors, cosmetics, and biomedicine, as well as ceramic and polymer composite applications.

It is believed that the synthesis of hybrid organic-inorganic bio-nanocomposites, biogenic inorganic materials, bioactive inorganic and hybrid materials; the identification of their properties; the understanding 


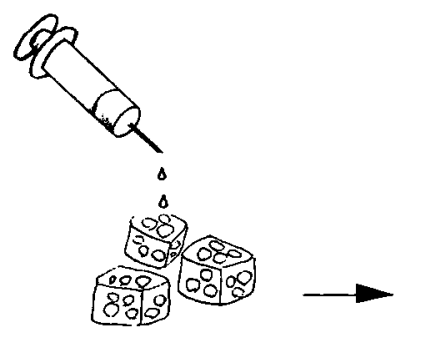

a

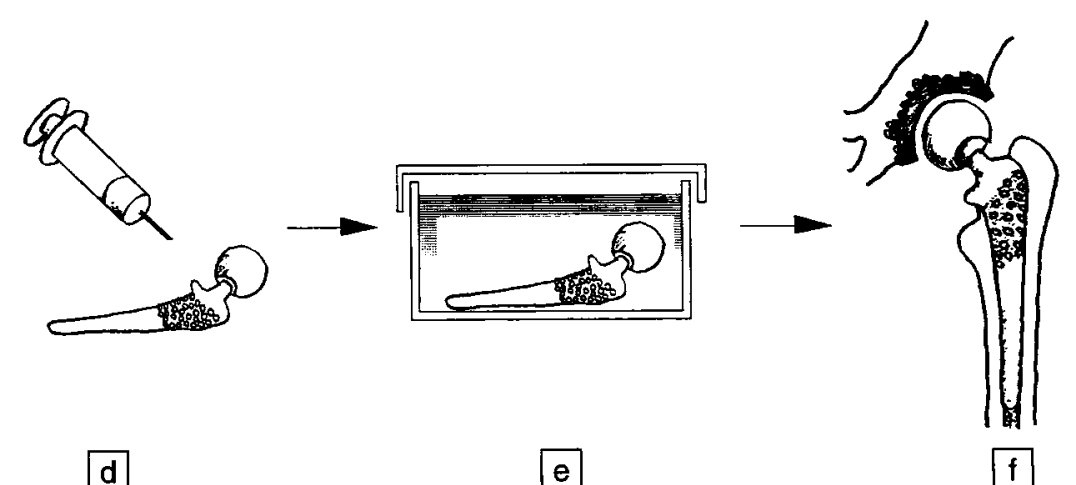

Figure 3. (a)-(c) Bone regeneration therapy using marrow mesenchymal cells for bone graft applications for increased bioactivity: (a) bone marrow cells from a syringe are combined with porous hydroxyapatite ( $H A p$, e.g., coralline apatite); (b) the marrow cell/HAp composite is cultured in an osteogenic medium; (c) after 2-3 weeks of culture, the cultured bone graft /HAp hybrid is inserted into a bone defect. (d)-(f) Application of bone regeneration therapy method using marrow mesenchymal cells to a macro textured orthopedic hip implant for increased bioactivity: (d) bone marrow cells are introduced into a joint prosthesis; (e) the prosthesis is cultured in an osteogenic medium; (f) after 2-3 weeks of culture, the prosthesis is implanted in a total hip replacement. (From Reference 15.)

of self-assembly and templated growth processing; and the engineered organization of solids at organic/inorganic interfaces will be the precursors of future nanosensors and nanomachines.

Biomimetic and bioinspired approaches to materials and templated growth of inorganic or hybrid networks using selfassembled hybrid organic-inorganic interfaces will also help to extend this promising domain. Chemically derived hybrid materials lie at the interface of the organicinorganic and biological realms. The main advantages in the use of these hybrids result from their high versatility, offering a wide range of possibilities to produce elaborate tailor-made materials in terms of chemical and physical properties. Moreover, these hybrid nanocomposites present the paramount advantage of facilitating integration, miniaturization, and multifunctionalization of the devices, opening a new range of opportunities for many applications.

In nature, many structures are produced by biomimetic processes. These are usually fine hybrid structures, and are hardly reproducible by current classical consolidation processes. However, it can be easily predicted that in due time, more and more inorganic/organic/biological hybrid materials incorporating bone morphogenic proteins and stem cells will be introduced, opening completely new perspectives in biomaterials production and application methods.

The current development of new novel nanoceramics is encouraging, and they can be used for a broad spectrum of medical applications such as implantable medical devices, tissue engineering, drug and gene delivery, imaging agents, materials for minimally invasive surgery, and biosensors. Bioceramics - and specifically, nanobioceramics-are fundamental to the design and development of a wide variety of medical devices and implants.

The scope for the application of nanoscale technologies to medical applications may cover the surgical, basic science, and engineering aspects of biomaterials and the relevant design and production characteristics of devices constructed from these biomaterials, as well as their clinical performance. Collaborative interdisciplinary research involving surgeons, materials scientists, biologists, physiologists, clinicians, engineers, and experts in any of the quantitative sciences is pertinent for the success of this exciting and promising field.

\section{References}

1. R.H. Doremus, J. Mater. Sci. 27 (1992) p. 285.

2. The World Orthopaedic Market 2000-2001

(Dorland's Biomedical/Knowledge Enterprises, Philadelphia, 2002).

3. L.L. Hench, Ann. N.Y. Acad. Sci. 523 (1988) p. 54 .

4. R.Z. LeGeros, Adv. Dent. Res. 2 (1988) p. 164.

5. T. Kokubo, H. Kushitani, Y. Ebisawa, T. Kitsugi, S. Kotani, K. Ohura, and T. Yamamuro, in Bioceramics, Vol. 1, edited by H. Oonishi, $\mathrm{H}$. Aoki, and K. Sawai (Ishiyaku EuroAmerica, Tokyo, 1989) p. 157.

6. K. deGroot, in Ceramics in Clinical Applications, edited by $\mathrm{P}$. Vincenzini (Elsevier, Amsterdam, 1987) p. 381.

7. H. Oonishi, I.C. Clarke, V. Good, H. Amino, M. Ueno, S. Masuda, K. Oomamiuda, H. Ishimaru, M. Yamamoto, and E. Tsuji, in Bioceramics 15 , edited by B. Ben-Nissan, D. Sher, and W. Walsh (Trans Tech Publications, UetikonZurich, 2003) p. 735.

8. B. Ben-Nissan, D.D. Green, G.S.K Kannangara, C.S. Chai, and A. Milev, J. Sol-Gel Sci. Technol. 21 (2001) p. 27.

9. B. Ben-Nissan, A. Milev, D. Green, R.M Conway, G.S.K. Kannangara, J. Russell, J. Hu, E Gillott, and C. Trefry, International Patent No. PCT -WO 02/40398 A1 (May 23, 2002).

10. T. Kokubo, in Bioceramics 15, edited by B. Ben-Nissan, D. Sher, and W. Walsh (Trans Tech Publications, Uetikon-Zurich, 2003) p. 523.

11. T. Kokubo, H.-M. Kim, M. Kawashita, and T. Nakamura, J. Aust. Ceram. Soc. 36 (1) (2000). p. 37.

12. G.J. Ehrhardt and D.E. Day, Nucl. Med. Biol. 14 (1987) p. 233.

13. M. Ikenaga, K. Ohura, T. Yamamuro, Y. Kotoura, M. Oka, and T. Kokubo, J. Orthop. Res. 11 (1993) p. 849

14. M. Kawashita, M. Tanaka, T. Kokubo, T. Yao, S. Hamada, and T. Shinjo, in Bioceramics 14 edited by S. Brown, I. Clarke, and P. Williams (Trans Tech Publications, Uetikon-Zurich, 2002) p. 645 .

15. T. Yoshikawa, T. Ohmura, Y. Sen, J. lida, Y. Takakura, I. Nonaka, and K. Ichijama, in Bioceramics 15 , edited by B. Ben-Nissan, D. Sher, and W. Walsh (Trans Tech Publications, UetikonZurich, 2003) p. 383.

16. T.J. Paunesku, T. Rajh, G. Wiederrecht, J. Mase, S. Vogt, N. Stojicevic, M. Protic, B. Lai, J. Oryhon, M. Thurnauer, and G. Woloschak, Nat. Mater. 2 (5) (2003) p. 343.

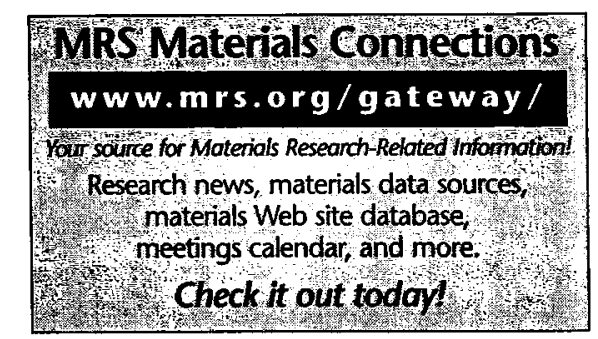


$\mathrm{MRS}$

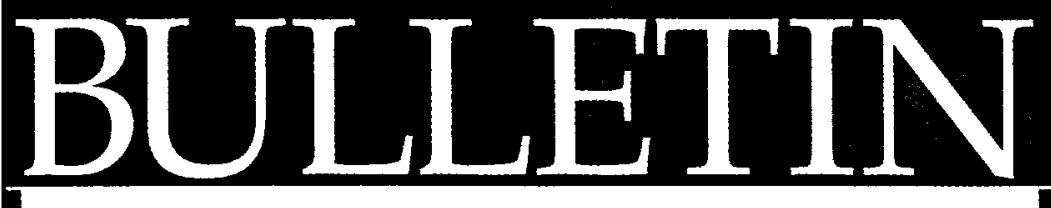

Serving the International Materials Research Community

January 2004, Volume 29, No. 1

A Publication of the Materials Research Society

\section{Toward Applications of Ceramic Nanostructures}

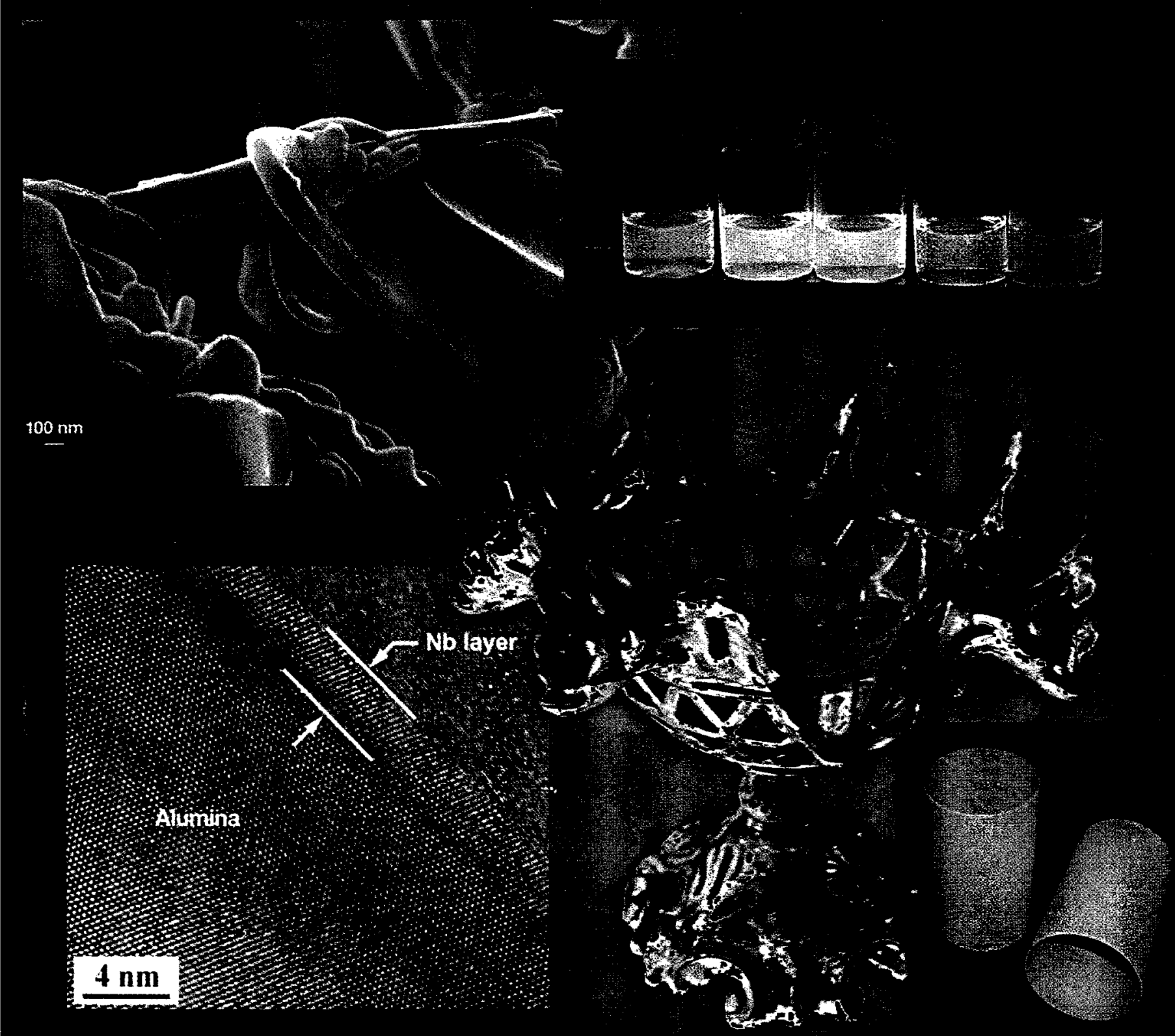

